\title{
Laser system of the autofluorescence polarimetry of cytological layers at an early stage of cancer detection
}

Yu. A. Ushenko

M. I. Sidor

M. V. Gritsyuk

G. D. Koval

O. Yu. Novakovskaya 


\title{
Laser system of the autofluorescence polarimetry of cytological layers at an early stage of cancer detection
}

\author{
Ushenko Yu.A. ${ }^{1}$, Sidor M.I. ${ }^{2}$, Gritsyuk M.V. ${ }^{3}$, Koval G.D. ${ }^{3}$, Novakovska O. Yu. ${ }^{3}$ \\ ${ }^{1}$ Correlation Optics Department, Chernivtsi National University, \\ 2 Kotsyubinsky Str., Chernivtsi, 58012, Ukraine \\ ${ }^{2}$ Optics and Publishing Department, Chernivtsi National University, \\ 2 Kotsyubinsky Str., Chernivtsi, 58012, Ukraine \\ ${ }^{3}$ Bukovinian State Medical University, Chernivtsi, 58000, Ukraine
}

a.dubolazov@,chnu.edu.ua

\begin{abstract}
A new information optical technique of diagnostics of the structure of polycrystalline films of blood plasma is proposed. The model of Mueller-matrix description of mechanisms of optical anisotropy of such objects as optical activity, birefringence, as well as linear and circular dichroism is suggested. The ensemble of informationally topical azimuthally stable Mueller-matrix invariants is determined. Within the statistical analysis of such parameters distributions the objective criteria of differentiation of films of blood plasma taken from healthy women and breast cancer patients were determined. From the point of view of probative medicine the operational characteristics (sensitivity, specificity and accuracy) of the information-optical method of Mueller-matrix mapping of polycrystalline films of blood plasma were found and its efficiency in diagnostics of breast cancer was demonstrated. Considered the prospects of applying the method in experimental medicine for differentiation of tissues of internal organs of healthy and diabetic rats.
\end{abstract}

Keywords: autofluorescence polarimetry, cytological layers, cancer detection.

\section{INTRODUCTION}

Nowadays in biological and medical investigations many practical techniques based on measurement and analysis of Mueller matrices are used [1,2]. In recent 10-15 years a separate approach - laser polarimetry of histological sections of biological tissues [3] - was formed in matrix optics. Within this approach the interactions between the set of statistical (moments of the $1^{\text {st }}-4^{\text {th }}$ orders) [4], and correlation (autocorrelation functions) [5] parameters characterizing Muellermatrix elements distribution and parameters of linear birefringence of human biological tissues were determined. On this basis the diagnostics of pathological changes of skin derma, epithelial and connective tissue of women's reproductive sphere, etc. was realized [6-8].

However, laser polarimetry techniques require further development and generalization. Firstly, not all elements of Mueller matrix prove to be convenient for characterizing biological samples. The reason of this is the azimuthal dependence of the majority of matrix elements - generally 12 of 16 elements change at rotation of the sample around the probing axis. Secondly, the spectrum of mechanisms of optical anisotropy of biological layers is not confined to linear birefringence only. Taking into consideration the impact of other mechanisms - circular birefringence, as well as linear and circular dichroism - appears to be topical in the aspect of enlarging the range of diagnostic techniques. Thirdly, there is a wide range of optically anisotropic biological objects, for which laser polarimetry techniques did not spread widely. Biological fluids - blood and its plasma, urine, bile, saliva and others - belong to them. The objects of this class are easily accessible and do not require the traumatic surgery of biopsy. Fourth, the methods of laser autofluorescence diagnosis is progressively developed.

Biosensing and Nanomedicine VII, edited by Hooman Mohseni, Massoud H. Agahi, Manijeh Razeghi, Proc. of SPIE Vol. 9166, 916617 · (C) 2014 SPIE · CCC code: 0277-786X/14/\$18 · doi: 10.1117/12.2061173 
This research is focused on the development of the method of "azimuthally stable" autofluorescence Muellermatrix mapping of optical anisotropy of blood plasma films in the task of breast cancer diagnostics.

\section{BRIEF THEORETICAL BACKGROUND}

The description of mechanisms of optical anisotropy characteristic of blood plasma films is based on the following model ideas [3,9]:

1. Crystals of basic proteins (polypeptide chains of amino acids of albumin and globulin) of blood plasma possess optical activity and are characterized by matrix operator

where

$$
\{\Omega\}=\left\|\begin{array}{cccc}
1 & 0 & 0 & 0 \\
0 & \omega_{22} & \omega_{23} & 0 \\
0 & \omega_{32} & \omega_{33} & 0 \\
0 & 0 & 0 & 1
\end{array}\right\|,
$$

$$
\omega_{i k}=\left\{\begin{array}{l}
\omega_{22}=\omega_{33}=\cos 2 \theta \\
\omega_{23}=-\omega_{32}=\sin 2 \theta
\end{array}\right.
$$

Here $\theta$ - rotation angle of polarization plane of the transformed light beam.

2. Polycrystalline networks of albumin and globulin in the plane of blood plasma smear possess the linear birefringence and linear dichroism. Optical manifestations of such mechanisms are completely described by Mueller matrices:

\subsection{Linear birefringence}

$$
\{D\}=\left\|\begin{array}{|cccc}
1 & 0 & 0 & 0 \\
0 & d_{22} & d_{23} & d_{24} \\
0 & d_{32} & d_{33} & d_{34} \\
0 & d_{42} & d_{43} & d_{44}
\end{array}\right\|,
$$

where

$$
d_{i k}=\left\{\begin{array}{l}
d_{22}=\cos ^{2} 2 \rho+\sin ^{2} 2 \rho \cos \delta ; \\
d_{23}=d_{32}=\cos 2 \rho \sin 2 \rho(1-\cos \delta) ; \\
d_{33}=\sin ^{2} 2 \rho+\cos ^{2} 2 \rho \cos \delta ; \\
d_{24}=-d_{42}=\sin 2 \rho \sin \delta ; \\
d_{34}=-d_{43}=\cos 2 \rho \sin \delta ; \\
d_{44}=\cos \delta .
\end{array}\right.
$$

Here $\rho$ - direction of optical axis of biological crystal, $\delta$ - phase shift between linearly polarized orthogonal components of light beam amplitude.

\subsection{Linear dichroism}

$$
\{\Psi\}=\left\|\begin{array}{cccc}
1 & \varphi_{12} & \varphi_{13} & 0 \\
\varphi_{21} & \varphi_{22} & \varphi_{23} & 0 \\
\varphi_{31} & \varphi_{32} & \varphi_{33} & 0 \\
0 & 0 & 0 & \varphi_{44}
\end{array}\right\|,
$$


where

$$
\varphi_{i k}=\left\{\begin{array}{l}
\varphi_{12}=\varphi_{21}=(1-\Delta \tau) \cos 2 \rho \\
\varphi_{13}=\varphi_{31}=(1-\Delta \tau) \sin 2 \rho \\
\varphi_{22}=(1+\Delta \tau) \cos ^{2} 2 \rho+2 \sqrt{\Delta \tau} \sin ^{2} 2 \rho \\
\varphi_{23}=\varphi_{32}=(1-\Delta \tau) \sin 2 \rho \\
\varphi_{33}=(1+\Delta \tau) \sin ^{2} 2 \rho+2 \sqrt{\Delta \tau} \cos ^{2} 2 \rho \\
\varphi_{44}=2 \sqrt{\Delta \tau}
\end{array}\right.
$$

Here $\Delta \tau=\frac{\tau_{x}}{\tau_{y}},\left\{\begin{array}{l}\tau_{x}=\tau \cos \rho \\ \tau_{y}=\tau \sin \rho\end{array}, \tau_{x}, \tau_{y}\right.$ - absorption coefficients of linearly polarized orthogonal components of light beam amplitude.

3. Availability of complex spiral-like protein molecular structures forms the circular dichroism. Optical manifestations of such configuration peculiarities are characterized by the Mueller matrix

where

$$
\{\Phi\}=\left\|\begin{array}{cccc}
1 & 0 & 0 & \phi_{14} \\
0 & \phi_{22} & 0 & 0 \\
0 & 0 & \phi_{33} & 0 \\
\phi_{41} & 0 & 0 & 1
\end{array}\right\|
$$

$$
\phi_{i k}=\left\{\begin{array}{l}
\phi_{22}=\phi_{33}=\frac{1-C^{2}}{1+C^{2}} \\
\phi_{14}=\phi_{41}= \pm \frac{2 C}{1+C^{2}} .
\end{array}\right.
$$

Here $C=\frac{g_{\otimes}-g_{\oplus}}{g_{\circledast}+g_{\oplus}}, g_{\circledast}, \quad g_{\oplus}$ - absorption indices of left- $(\otimes)$ and right-hand $(\oplus)$ circularly polarized components of light beam amplitude.

Considering the influence of all mechanisms of optical anisotropy Mueller matrix of blood plasma can be written as follows

$$
\{M\}=\{\Omega\}\{D\}\{\Psi\}\{\Phi\}=\left\|\begin{array}{cccc}
1 & M_{12} & M_{13} & M_{14} \\
M_{21} & M_{22} & M_{23} & M_{24} \\
M_{31} & M_{32} & M_{33} & M_{34} \\
M_{41} & M_{42} & M_{43} & M_{44}
\end{array}\right\| M_{i k} \neq 0 .
$$

It is shown in [10-25] that the following elements of the matrix $\{M\}$ and their combinations are azimuthally stable, independent of the rotation angle $(\Theta)$ of a sample

$$
\left\{\begin{array}{l}
M_{11}(\Theta)=\text { const } \\
M_{14}(\Theta)=\text { const } \\
M_{41}(\Theta)=\text { const } \\
M_{44}(\Theta)=\text { const }
\end{array}\right.
$$




$$
\left\{\begin{array}{l}
{\left[M_{22}+M_{33}\right](\Theta) \equiv \Sigma M_{22 ; 33}(\Theta)=\text { const }} \\
{\left[M_{23}-M_{32}\right](\Theta) \equiv \Delta M_{23 ; 32}(\Theta)=\text { const }}
\end{array}\right.
$$

The analysis of the rotation invariants (10), (11) reveals their individual "information" loading. Let us consider this statement in detail. In the visible part of the spectrum the manifestations of phase anisotropy predominate - linear and circular birefringence. Extreme values of the parameters of linear $(\Delta \tau)$ and circular dichroism $(C)$ of proteins of albumin and globulin are in the ultraviolet domain. In other words

$$
\delta, \theta>\Delta \tau, C .
$$

Considering (12), the matrix operator (9) can be rewritten as

$$
\{M(\rho, \delta, \theta)\}=\left\|\begin{array}{|cccc}
1 & 0 & 0 & 0 \\
0 & \left(d_{22} \omega_{22}+d_{23} \omega_{32}\right) & \left(d_{22} \omega_{23}+d_{23} \omega_{33}\right) & d_{24} \\
0 & \left(d_{32} \omega_{22}+d_{33} \omega_{32}\right) & \left(d_{32} \omega_{23}+d_{33} \omega_{33}\right) & d_{34} \\
0 & \left(d_{42} \omega_{22}+d_{43} \omega_{32}\right) & \left(d_{42} \omega_{23}+d_{43} \omega_{33}\right) & d_{44}
\end{array}\right\| \equiv\left\|\begin{array}{cccc}
1 & 0 & 0 & 0 \\
0 & M_{22} & M_{23} & M_{24} \\
0 & M_{32} & M_{33} & M_{34} \\
0 & M_{42} & M_{43} & M_{44}
\end{array}\right\| .
$$

It follows from (13) that the values of Mueller-matrix rotation invariants $M_{44}(\Theta), \frac{\Delta M_{23 ; 32}(\Theta)}{\sum M_{22 ; 33}(\Theta)}$ are generally determined by the parameters of linear $(\delta)$ and circular $(\theta)$ birefringence

$$
\left\{\begin{array}{l}
M_{44} \sim \cos \delta \\
\frac{\Delta M_{23 ; 32}}{\sum M_{22 ; 33}} \sim \tan \theta .
\end{array}\right.
$$

Despite the lower level of manifestations of the effects of anisotropic absorption of laser radiation (relations (5)(8)) some Mueller matrix elements appear to be sensitive to such mechanisms. At the presence of linear dichroism $(\Delta \tau)$ the matrix operator (9) is transformed into expression

$$
\{M(\rho, \delta, \theta, \Delta \tau)\}=\left\|\begin{array}{cccc}
1 & \left(\varphi_{12} t_{22}+\varphi_{13} t_{32}\right) & \left(\varphi_{12} t_{23}+\varphi_{13} t_{33}\right) & \left(\varphi_{12} t_{24}+\varphi_{13} t_{34}\right) \\
\varphi_{21} & \left(\varphi_{22} t_{22}+\varphi_{23} t_{32}\right) & \left(\varphi_{22} t_{23}+\varphi_{32} t_{33}\right) & \left(\varphi_{22} t_{24}+\varphi_{32} t_{34}\right) \\
\varphi_{31} & \left(\varphi_{32} t_{22}+\varphi_{33} t_{32}\right) & \left(\varphi_{32} t_{23}+\varphi_{33} t_{33}\right) & \left(\varphi_{32} t_{24}+\varphi_{33} t_{34}\right) \\
0 & \varphi_{44} t_{42} & \varphi_{44} t_{43} & \varphi_{44} t_{44}
\end{array}\right\| .
$$

Comparison of (13) and (15) shows that the condition $M_{14}(\Theta) \neq 0$ is the criterion of the presence of linear dichroism

$$
M_{14}=\varphi_{12} d_{24}+\varphi_{13} d_{34}=(1-\Delta \tau) \sin \delta
$$

Circular dichroism $(C)$ is manifested unlike zero of rotation invariant $M_{41}\left(\lambda_{2}\right) \neq 0$

$$
M_{41}=\varphi_{44} d_{44} c_{41}=4 \sqrt{\Delta \tau} \cos \delta \frac{C}{1+C^{2}} .
$$


Thus, having measured the parameters distributions $\left(q \equiv\left\{\begin{array}{l}M_{14 ; 41 ; 44}(m \times n) ; \\ \Delta M_{i k}=\frac{\Delta M_{23 ; 32}}{\sum M_{22 ; 33}}(m \times n) \text {, one can obtain azimuthally }\end{array}\right.\right.$ stable information about optical anisotropy of proteins of blood plasma film.

\section{INVESTIGATION TECHNIQUE AND PROCESSING OF MUELLER-MATRIX IMAGES}

The measurements of coordinate distributions of Mueller-matrix elements were performed in the setup of the standard Stokes-polarimeter [3]. For excitation we used autofluorescence emission of blue $(\lambda=0,405 \mu m)$ solid-state laser.

The Stokes-vector parameters $S_{i=2 ; 3 ; 4}^{0 ; 45 ; 9}$ were measured in the points $(m \times n)$ of the digital image

$$
\left\{\begin{array}{l}
S_{i=2}^{0 ; 45 ; 90 ; \otimes}=I_{0}^{0 ; 45 ; 90 ; \otimes}-I_{90}{ }^{0 ; 45 ; 90 ; \otimes} ; \\
S_{i=3}^{0 ; 45 ; 90 ; \otimes}=I_{45}^{0 ; 45 ; 90 ; \otimes}-I_{135}^{0 ; 45 ; 90 ; \otimes} \\
S_{i=4}^{0 ; 45 ; 90 ; \otimes}=I_{\otimes}^{0 ; 45 ; 90 ; \otimes}-I_{\oplus}^{0 ; 45 ; 90 ; \otimes} .
\end{array}\right.
$$

Here $I_{0 ; 90 ; 45 ; 135 ; \otimes ; \oplus}^{0 ; 45 ; 9 ; \otimes}$ - intensities of linearly $\left(0^{0} ; 90^{0} ; 45^{0} ; 135^{\circ}\right)$, right- $(\otimes)$ and left- $(\oplus)$ circularly polarized components of the filtered (by means of polarizer 9 and quarter-wave plate 8 ) laser radiation.

Further the "informationally topical" parameters (10),(11) were calculated using the algorithm

$$
\left\{\begin{array}{l}
M_{44}=S_{4}^{\otimes}-0,5\left(S_{4}^{0}+S_{4}^{90}\right) ; \\
\sum M_{22 ; 33}=M_{22}+M_{33}=0,5\left(S_{2}^{0}-S_{2}^{90}\right)+S_{3}^{45}-0,5\left(S_{3}^{0}+S_{3}^{90}\right) ; \\
\Delta M_{23 ; 32}=M_{23}-M_{32}=S_{2}^{45}-0,5\left(S_{2}^{0}+S_{2}^{90}\right)-0,5\left(S_{3}^{0}-S_{3}^{90}\right) .
\end{array}\right.
$$

For objective assessment of coordinate distributions $q \equiv\left\{\begin{array}{l}M_{14 ; 41 ; 4}(m \times n) ; \\ \frac{\Delta M_{23 ; 32}}{\sum M_{22 ; 33}}(m \times n)\end{array}\right.$ we applied the synthesis of traditional methods of statistical and correlation analysis.

A set of statistical moments of the $1^{\text {st }}-4^{\text {th }}$ orders characterizing distributions $q$ was calculated using the algorithms

$$
Z_{1}=\frac{1}{N} \sum_{j=1}^{N}|q| ; Z_{2}=\sqrt{\frac{1}{N} \sum_{j=1}^{N}(q)_{j}{ }^{2}} ; Z_{3}=\frac{1}{Z_{2}{ }^{3}} \frac{1}{N} \sum_{j=1}^{N}(q)_{j}^{3} ; Z_{4}=\frac{1}{Z_{2}{ }^{4}} \frac{1}{N} \sum_{j=1}^{N}(q)_{j}{ }^{4} .
$$

$N$ - number of pixels of CCD-camera.

\section{ANALYSIS AND DISCUSSION OF EXPERIMENTAL DATA}

Optically thin (attenuation factor $\tau<0.1$ ) donors' blood plasma films dried at room temperature (group $1-25$ patients) and those of breast cancer patients (group $2-25$ patients) were used as objects of investigation. Figures $1-4$ present the series of experimentally measured Mueller-matrix images $M_{i k}(m \times n)$ (relations (19)). 


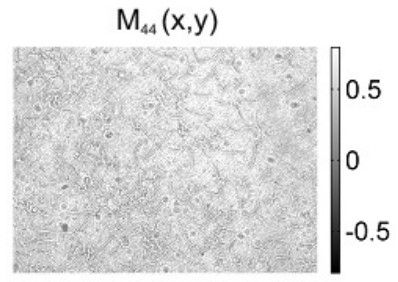

(a)

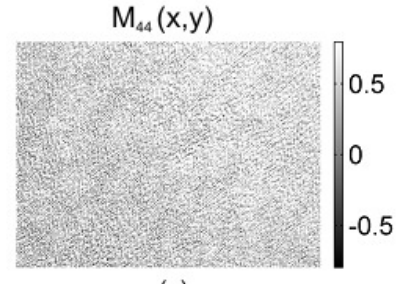

(c)
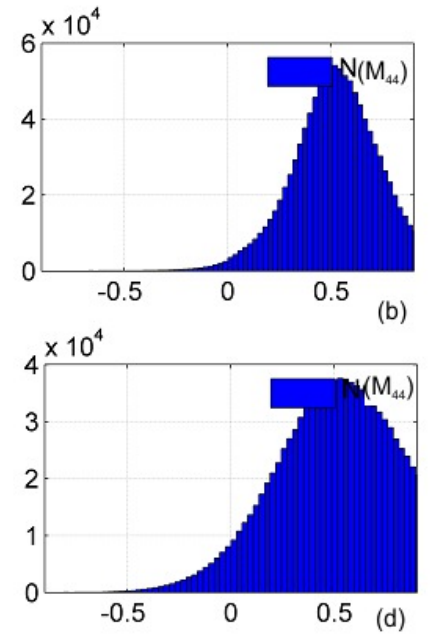

Fig. 1. Coordinate distributions of Mueller-matrix rotation invariant $M_{44}$, characterizing linear birefringence of films of donor's blood plasma ((a) - (b)) and of cancer patient ((c) (d))

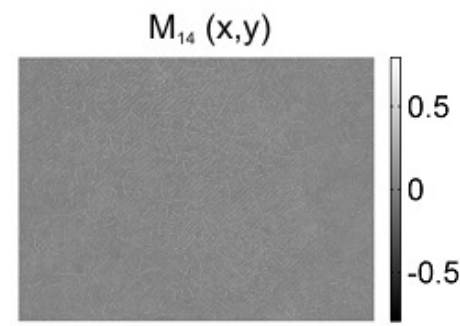

(a)

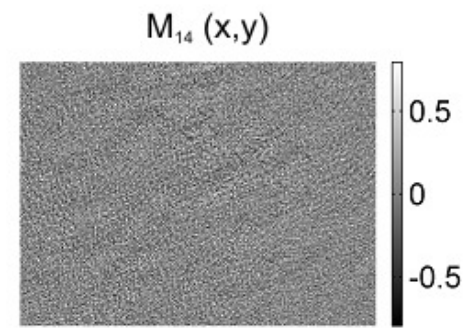

(c)
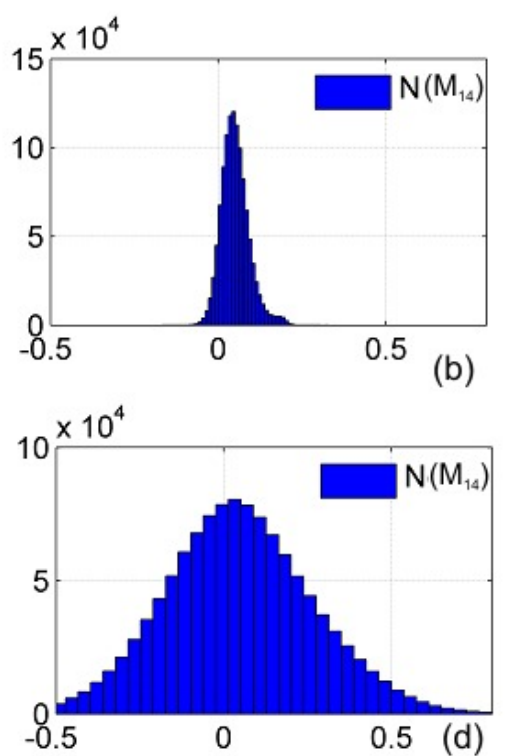

Fig. 2. Coordinate distributions of Mueller-matrix rotation invariant $M_{14}$, characterizing linear dichroism of films of donor's blood plasma ((a) - (b)) and of cancer patient ((c) - (d)). 


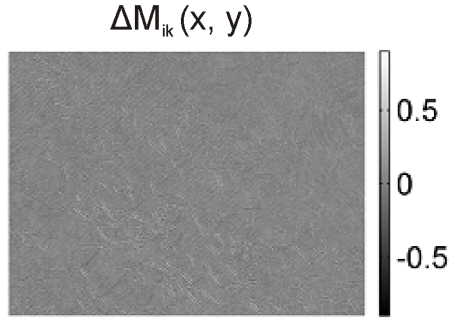

(a)

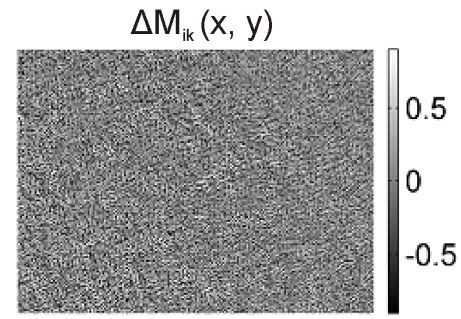

(c)
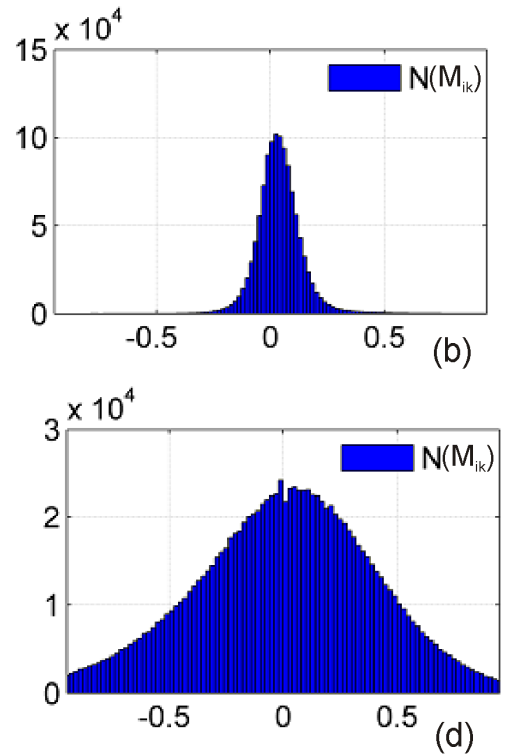

Fig. 3. Coordinate distributions of Mueller-matrix rotation invariant $\Delta M_{i k}$, characterizing circular birefringence of films of donor's blood plasma ((a) - (b)) and of cancer patient ((c) - (d)).

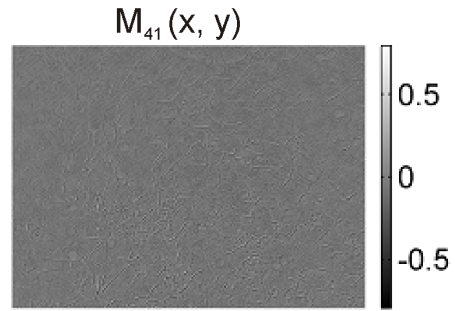

(a)

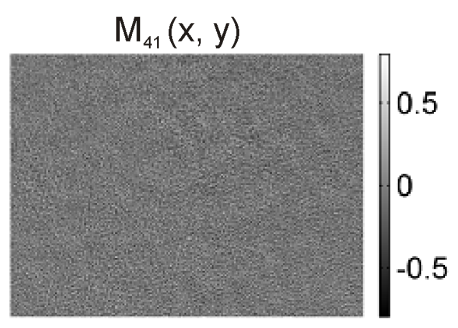

(c)
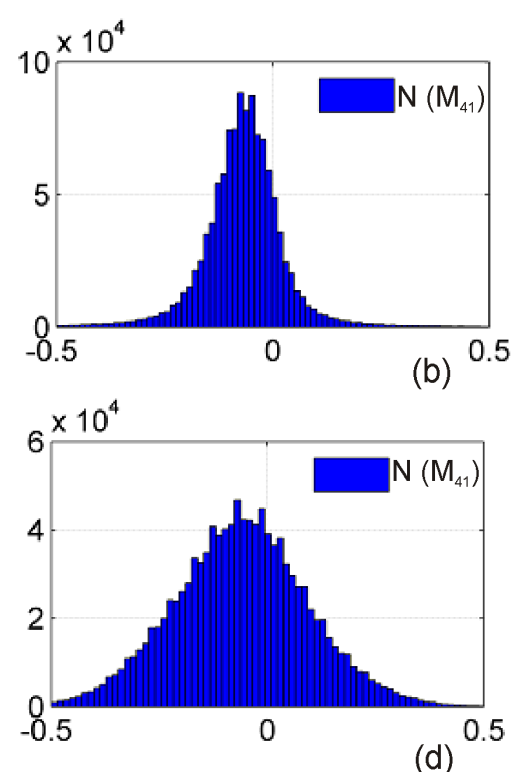

Fig. 4. Coordinate distributions of Mueller-matrix rotation invariant $M_{41}$, characterizing circular dichroism of films of donor's blood plasma ((a) - (b)) and of cancer patient ((c) - (d)).

The comparative analysis of the data obtained showed:

- Linear birefringence (Fig. 1). Distributions $M_{44}(m \times n)$ found for samples of both types are characterized by commensurable range of values change and rather identical coordinate structure. This fact can be related to 
that close in values to phase shifts $\delta$ determined by simultaneous contribution of the value of birefringence and transverse sizes of albumin crystals.

- Linear dichroism (Fig. 2). It is determined that protein network of donor's blood plasma film has greater level of linear dichroism in comparison with similar sample of cancer patient. The differences found between Mueller-matrix images $M_{14}(m \times n)$ can be related to the differences in concentration of basic proteins of blood plasma. For the sample from group 1 greater concentration of albumin is typical. It is observed in formation of the network of spatially oriented lathlike crystals. On the contrary, the increase of concentration of optically active globulin leads to formation of restructured network with minimal linear dichroism.

- Circular birefringence (Fig. 3). It is defined that distributions $\Delta M_{i k}$ of the film of blood plasma of cancer patients exceed the similar dependences for the samples from group 1.

- Circular dichroism (Fig. 4). Optically the anisotropic manifestations of tertiary structure of albumin and globulin molecules are more vividly manifested for polycrystalline network of the film of blood plasma of a healthy person. The range of changes of the value of matrix element $M_{41}$ of the sample from group 1 are by $2-3$ times greater than in Mueller-matrix image of the sample from group 2.

The results of quantitative statistical (relations (19)) and correlation (relations (20), (21)) analysis of a series of Mueller-matrix rotational invariants of two groups of blood plasma films are illustrated by the data presented in Table 1.

The most sensitive parameters of differentiation of optically anisotropic networks of blood plasma proteins of both groups are highlighted by grey color in Table 1 .

For possible clinical application of Mueller-matrix mapping within the investigated groups of samples the operational characteristics typical for probative medicine are: sensitivity $\left(S e=\frac{a}{a+b} 100 \%\right)$, specificity $\left(S p=\frac{c}{c+d} 100 \%\right)$ and accuracy $\left(A c=\frac{S e+S p}{2}\right)$, where $a$ and $b-$ the number of correct and wrong diagnoses within group $1 ; c$ and $d$ - the same within group 2 - Table 2.

Table 1. Statistical ( $Z_{i=1 ; 2 ; 3 ; 4}$ ) moments of distributions of Mueller-matrix invariants of films of blood

\begin{tabular}{|c|c|c|c|c|c|c|c|c|}
\hline \multirow{2}{*}{$\begin{array}{l}\text { Parameter } \\
\text { s }\end{array}$} & \multicolumn{2}{|c|}{$M_{44}$} & \multicolumn{2}{|c|}{$M_{14}$} & \multicolumn{2}{|c|}{$\Delta M_{i k}$} & \multicolumn{2}{|c|}{$M_{41}$} \\
\hline & Normal & Cancer & Normal & Cancer & Normal & Cancer & Normal & Cancer \\
\hline$Z_{1}$ & $\begin{array}{c}0,34 \pm 0,06 \\
1\end{array}$ & $0,26 \pm 0,05$ & $\frac{0,18 \pm 0}{8}$ & $0,11 \pm 0,01$ & $0,12 \pm 0,02$ & $\frac{0,18 \pm 0,03}{8}$ & $0,18 \pm 0,01$ & $\frac{0,09 \pm 0,01}{1}$ \\
\hline$Z_{2}$ & $0,16 \pm \frac{0,01}{8}$ & $0,19 \pm \frac{0}{4} 0,02$ & $0,22 \pm 0,03$ & $0,15 \pm 0,01$ & $0,15 \pm 0,01$ & $0,11 \pm 0,02$ & $0,13 \pm \frac{0,04}{6}$ & $0,11 \pm 0,01$ \\
\hline$Z_{3}$ & $0,23 \pm 0,04$ & $\begin{array}{c}0,31 \pm 0,06 \\
1\end{array}$ & $0,78 \pm 0,14$ & $1,31 \pm 0,39$ & $0,79 \pm 0,12$ & $0,53 \pm 0,05$ & $1,27 \pm 0,15$ & $2,81 \pm 0,53$ \\
\hline$Z_{4}$ & $0,29 \pm 0,02$ & $0,22 \pm 0,03$ & $0,88 \pm 0,11$ & $2,14 \pm 0,47$ & $0,68 \pm 0,16$ & $0,44 \pm \frac{0,06}{5}$ & $1,02 \pm 0,22$ & $2,59 \pm 0,73$ \\
\hline
\end{tabular}

Table 2. Operational characteristics of Mueller-matrix mapping techniques

\begin{tabular}{|c|c|c|c|c|}
\hline Parameters & $M_{44}$ & $M_{14}$ & $\Delta M_{i k}$ & $M_{41}$ \\
\hline$S e\left(Z_{i}\right), \%$ & 75 & 86 & 77 & 88 \\
\hline$S p\left(Z_{i}\right), \%$ & 66 & 77 & 74 & 71 \\
\hline$A c\left(Z_{i}\right), \%$ & 71 & 79 & 82 & 86 \\
\hline
\end{tabular}


Thus, the statistical and correlation analyses of Mueller-matrix invariants characterizing polarization manifestations of linear dichroism, circular birefringence and circular dichroism appeared to be efficient in the task of differential diagnostics of breast cancer.

This method was tested in the problem of differentiation of histological sections of the internal organs of healthy and diabetic rats (liver, spleen, kidney and pancreas) - Table 3 .

Table 3. Operational characteristics of Mueller-matrix mapping techniques

\begin{tabular}{|c|c|c|c|c|}
\hline Parameters & liver & spleen & kidney & pancreas \\
\hline$S e\left(Z_{i}\right), \%$ & 74 & 81 & 87 & 68 \\
\hline$S p\left(Z_{i}\right), \%$ & 69 & 74 & 78 & 73 \\
\hline$A c\left(Z_{i}\right), \%$ & 72 & 77 & 84 & 71 \\
\hline
\end{tabular}

\section{CONCLUSIONS}

1. The Mueller-matrix invariants characterizing polarization manifestations of partial mechanisms of optical anisotropy - linear birefringence, linear dichroism, circular birefringence and circular dichroism of polycrystalline film of blood plasma are determined.

2. Within the statistical approach the interconnections between the set of moments of the $1^{\text {st }}-4^{\text {th }}$ orders and peculiarities of optically anisotropic networks of proteins of blood plasma of healthy people and cancer patients are defined.

3. The efficiency of the technique of azimuthally invariant Mueller-matrix mapping of polycrystalline films of blood plasma in the task of breast cancer diagnostics differentiation of changes of optical anisotropy of the tissue internal organs rat are demonstrated.

\section{REFERENCES}

[1]. Ushenko, A.,G., Pishak, V.,P., "Laser Polarimetry of Biological Tissue: Principles and Applications," Handbook of Coherent-Domain Optical Methods: Biomedical Diagnostics, Environmental and Material Science 1, p.p. 93-138, (2004).

[2]. Angelsky, O.,V., Ushenko A.,G., Ushenko, Yu.A., Pishak, V.P., Peresunko, A.P., "Statistical, Correlation and Topological Approaches in Diagnostics of the Structure and Physiological State of Birefringent Biological Tissues," Handbook of Photonics for Biomedical Science, p.p. 283-322 (2010).

[3]. Ushenko, Y.A., Boychuk, T.M., Bachynsky, V.T., Mincer, O.P., "Diagnostics of Structure and Physiological State of Birefringent Biological Tissues: Statistical, Correlation and Topological Approaches," Handbook of Coherent-Domain Optical Methods, p.p. 107-148 (2013).

[4]. Ushenko, Yu.A., Bodnar, G.B., Koval, G.D., "Classifying optical properties of surface-and bulk-scattering biological layers with polarization singular states," Journal of Innovative Optical Health Sciences 6, p.p. 1350018 (2013).

[5]. Ushenko, Yu.A., "Statistical structure of polarization-inhomogeneous images of biotissues with different morphological structures,” Ukr. J. Phys. Opt. 6, p.p. $63-70$ (2005).

[6]. Ushenko, Yu.A., Peresunko, A.P., Baku, B.A., "A New Method of Mueller-Matrix Diagnostics and Differentiation of Early Oncological Changes of the Skin Derma," Advances in Optical Technologies 2010, p.p. 952423 (2010).

[7]. Ushenko, Yu.A., Telenga, O.I., Peresunko, A.P. , Numan, O.K., "New parameter for describing and analyzing the optical-anisotropic properties of biological tissues," J. Innov. Opt. Health Sci. 4, p.p. 463-475 (2011).

[8]. Ushenko, Yu.A., "The feasibilities of using the statistical, fractal and singular processing of hominal blood plasma phase images during the diagnostics and differentiation of mammary gland pathological states," J. Innov. Opt. Health Sci. 5, p.p. 1150001 (2012).

[9]. Savenkov, S.N., Marienko, V.V., Oberemok, E.A., Sydoruk, O.I., "Generalized Matrix Equivalence Theorem for Polarization Theory," Phys. Rev. E. 74, p.p. 605-607 (2006). 
[10]. Shribak, M. Oldenbourg, R., "Techniques for Fast and Sensitive Measurements of Two-Dimensional Birefringence Distributions," Appl. Opt. 42, p.p. 3009-3017 (2003).

[11]. Smith, M.H, "Interpreting Mueller matrix images of tissues," Proc. SPIE 4257, p.p. 82-89 (2001).

[12]. Lu S., Chipman, R. A., "Interpretation of Mueller matrices based on polar decomposition," J. Opt. Soc. Am. A 13, p.p. 1106-1113 (1996).

[13]. Angelsky, O.,V., Polyanskii, P.,V., Felde, C.,V., "The emerging field of correlation optics," Optics and Photonics News 23(4), p.p.25-29 (2012).

[14]. Angelsky, O., V., Ushenko, A., G., Burcovets, D., N., Ushenko, Yu., A., "Polarization visualization and selection of biotissue image two-layer scattering medium," J. Biomed. Opt. 10(1), P. 014010 (2005).

[15]. Bekshaev, A., Y., Angelsky, O., V., Hanson, S., G., Zenkova, C., Y., "Scattering of inhomogeneous circularly polarized optical field and mechanical manifestation of the internal energy flows," Phys. Rev. A. (86), p.p. 023847 (2012).

[16]. Angelsky, O.,V., Bekshaev, A.,Ya., Maksimyak, P.,P., Maksimyak, A.,P., Hanson, S.,G., Zenkova, C.,Yu., "Self-diffraction of continuous laser radiation in a disperse medium with absorbing particles," Optics Express 21(7), p.p. 8922-8938 (2013).

[17]. Angelsky, O., V., Hanson, S.,G., Maksimyak, A.,P., Maksimyak, P.,P., "On the feasibility for determining the amplitude zeroes in polychromatic fields," Optics Express 13(12), p.p. $4396-4405$ (2005).

[18]. Angelsky, O.V., Besaha, R.N., Mokhun, I.I. "Appearance of wavefront dislocations under interference among beams with simple wavefronts," Optica Applicata 27(4), p.p. 272-278 (1997).

[19]. Angelsky, O.,V., Bekshaev, A.,Ya., Maksimyak, P., P., Maksimyak, A., P., Hanson, S., G., Zenkova, C.,Yu., "Orbital rotation without orbital angular momentum: mechanical action of the spin part of the internal energy flow in light beams," Optics Express, 20(4), p.p.3563-3571 (2012).

[20]. Angelsky, O.,V., Demianovsky, G.,V., Ushenko, A.,G., Burkovets, D.,N., Ushenko, Y.,A., "Wavelet analysis of two-dimensional birefringence images of architectonics in biotissues for diagnosing pathological changes," Journal of Biomedical Optics 9 (4), p.p. 679-690 (2004).

[21]. Angel'skiǐ, O.,V., Ushenko, A.,G., Ermolenko, S.,B., Burkovets,D.,N., Ushenko, Yu.,A., Pishak, O.,V., "Polarization-Based Visualization of Multifractal Structures for the Diagnostics of Pathological Changes in Biological Tissues," Optics and Spectroscopy (English translation of Optika i Spektroskopiya) 89 (5), p.p. 799-804 (2000).

[22]. Ushenko, Y.,O., TOMKA, Y.,Y., Dubolazov, O.,V., BALANETSKA, V.,O., Karachevtsev, A.,V., ANGELSKY, A.,P., "Wavelet-analysis for Laser Images of Blood Plasma," AECE-Advances in Electrical and Computer Engineering, 11(2), (2011).

[23]. Bachinsky, V.,T., Ushenko, Yu.,O., Tomka, Yu.,Ya., Dubolazov, O.,V., Balanets'ka, V.,O., Karachevtsev, A.,V., "Wavelet analysis for polarization maps of networks formed by liquid biological crystals in blood plasma: statistical and fractal approaches," Semiconductor Physics, Quantum Electronics\& Optoelectronics 13(2), P. 189-201 (2010).

[24]. Ushenko, Yu., A, Tomka, Yu., Ya., Dubolazov, A.,V., Balanetskaya, V.,A., Unguryan, V.,P.,, Zabolotna, N.,I., Oleinichenko, B.,P., "Mueller-matrix diagnostics of optical properties inherent to polycrystalline networks of human blood plasma," Semiconductor Physics, Quantum Electronics \& Optoelectronics, 14(1), p.p. 98-105 (2011).

[25]. Ushenko, Yu., O., Dubolazov, O., V., Karachevtsev, A., O., Gorsky, M., P., Marchuk, Yu., F., "Wavelet analysis of Fourier polarized images of the human bile," Appl. Opt. 51, p.p.133-C139 (2012) 